\title{
Unmasking of a second atrioventricular accessory connection by adenosine in a child with a long $\mathrm{RP}^{\prime}$ reentrant tachycardia
}

Matthew M Wienecke, Christopher L Case, Paul C Gillette

\begin{abstract}
A second unidirectional, retrograde accessory atrioventricular pathway was unmasked by adenosine during the intracardiac evaluation of a child with a reentrant long $\mathbf{R P}^{\prime}$ tachycardia. This case is further evidence of the value of adenosine during the evaluation of these types of tachycardias.
\end{abstract}

(Br Heart J 1992;68:216-7)

Medical University of South Carolina, Division of Pediatric Cardiology,

Charleston, South

Carolina, USA

M M Wienecke

C L Case

P C Gillette

Correspondence to

Dr Matthew M Wienecke, Medical University of South Carolina, Children's Heart Charleston, SC 29425, USA

\section{Adenosine generally terminates orthodromic supraventricular tachycardia through its anti- dromotropic effects on the anterograde limb (that is, the atrioventricular node) of reentrant atrioventricular tachycardias. ${ }^{1-3}$ However, in patients with reentrant forms of long $R P^{\prime}$ tachycardia, adenosine also has anti- dromotropic effects on the retrograde limb. ${ }^{34}$ These anti-dromotropic effects occur because of the postulated "atrioventricular node like"}
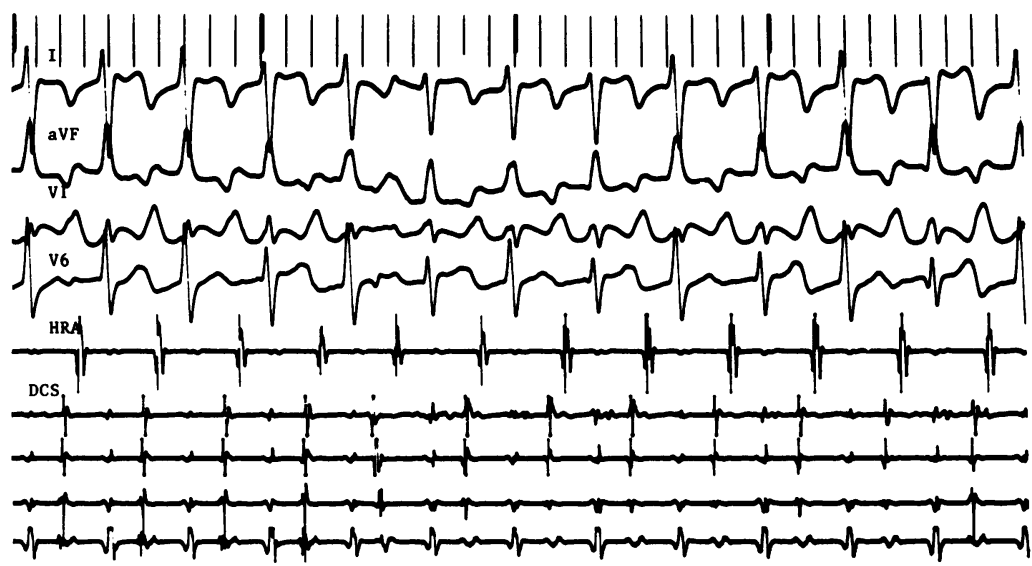

ग 1. (2)

Figure 1 Surface electrocardiographic leads I, aVF, V1, and V6 recorded simultaneously with high right atrial, six coronary sinus, two His-bundle, and one right ventricular apex electrocardiograms after adenosine administration during tachycardia. The tachycardia cycle length is $320 \mathrm{~ms}$. After the first four $Q R S$ complexes the site of earliest retrograde atrial activation was pair 4 of the coronary sinus (CS) catheter. After the fifth $Q R S$ complex the atrial activation sequence changed so that the distal $C S$ pair was earliest. This second atrial activation sequence after transiant retrograde conduction block of the primary limb suggests a second retrograde pathway. HRA, High right atrial; $D C S$, distal coronary sinus; $P C S$, proximal coronary sinus; $H B E, H i s$ bundle electrogram; $R V A$, right ventricular apex. properties of the retrograde limb. We report a case in which adenosine by producing transient retrograde conduction block unmasked a second unidirectional retrograde atrioventricular accessory pathway.

\section{Case report}

Intermittent tachycardia was first diagnosed in a four year old white girl six months before admission. She had been symptom free apart from a tendency to tire quickly with exercise. Her physical examination was remarkable only for the presence of an irregular heart rate. Her surface electrocardiogram showed long $\mathbf{R P}^{\prime}$ tachycardia with a heart rate of 165 beats per minute, an $R P / R R$ ratio of $61 \%$, and negative $P$ waves in leads I-III and aVF. A 24 hour ambulatory monitor showed $22 \%$ tachycardia. Her chest $x$ ray and echocardiogram were normal.

Because the tachycardia was resistant to digoxin, propranolol, and verapamil, an intracardiac electrophysiological evaluation with pre-ablation mapping was performed. This study included both atrial and ventricular protocols at rest and during autonomic blockade. No pre-excitation was noted during sinus or paced atrial rhythms. During ventricular (VOO) pacing, a unidirectional retrograde accessory pathway was mapped off the septum in the posterior wall of the left atrium. A retrograde Wenckebach block was elicited during VOO pacing at a cycle length of $290 \mathrm{~ms}$, suggesting that this limb had decremental properties. By producing a transient retrograde conduction block, the administration of adenosine during tachycardia provided further evidence about the decremental properties of this pathway. Furthermore, during the single beat of retrograde conduction block produced by adenosine a second retrograde atrial activation sequence was unmasked (fig 1). This finding implied a second unidirectional retrograde pathway located in the lateral left atrium. After autonomic blockade, this unmasked retrograde atrial activation sequence was again noted when a retrograde block was produced by introducing single premature ventricular contractions into VOO pacing.

Because of these findings the patient had surgical ablation. Her intraoperative epicardial map confirmed both pathways (fig 2 ). The incision was extended well into the lateral free wall of her left atrium. Postoperational wire electrode studies showed the absence of 


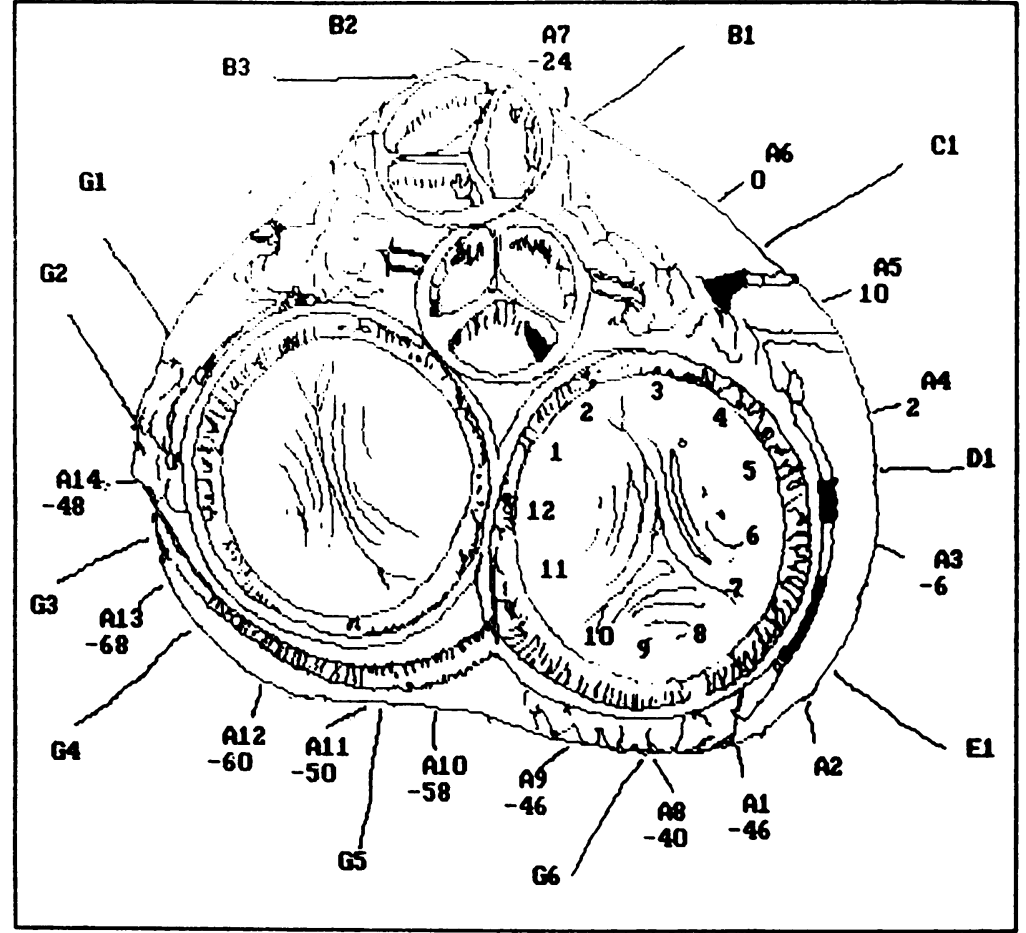

Figure 2 Intraoperative map during supraventricular tachycardia. The illustration is a cross section of the heart as viewed from above. The mitral valve is to the left, the tricuspid valve to the right, and the pulmonary valve most anterior. The letter-number combinations (for example, G4) represent specific mapping sites. The negative numbers represent atrial activation as referenced to a right atrial appendage electrogram.

Therefore, the more negative the number the earlier the atrial activation sequence. There are two non-contiguous areas of early atrial activation: A13 (left lateral free wall) and A10 (left posterior free wall).
Because the conduction of the primary unidirectional retrograde limb of this child's tachycardia, had "atrioventricular node like" properties it was transiently blocked by adenosine. And this unmasked, a second unidirectional retrograde accessory atrioventricular pathway (fig 1). Although it is impossible to exclude a premature atrial contraction as the cause of this second atrial activation sequence, the reproducibility of this second activation sequence during catheterisation and the demonstration of two non-contiguous areas of early atrial activation on an intraoperative map are strong evidence of a second pathway.

Adenosine not only gave information about the decremental properties of the retrograde limb but helped to establish the presence of a second accessory pathway. The combination of these diagnoses was important when this child was assessed for surgical or radiofrequency catheter ablation. As has recently been reported by Bharati et al these pathways with "atrioventricular node like" properties may in fact be left sided atrioventricular nodes that may prove difficult to ablate by catheter techniques. 5 This factor, combined with evidence of a second pathway, led us to favour surgical ablation.

The usefulness of adenosine for the evaluation of patients with long $R P^{\prime}$ reentrant tachycardias is becoming more apparent. Adenosine is not only a powerful therapeutic agent but also an important diagnostic tool.

retrograde conduction. She has remained tachycardia free during four months of follow up.

Although the location of the retrograde limb of reentrant long $\mathbf{R P}^{\prime}$ tachycardias, and therefore the site of earliest retrograde atrial activation, may vary with the specific type of tachycardia (that is, "fast-slow" form of atrioventricular node reentrant tachycardia versus the permanent form of junctional reciprocating tachycardia versus accessory atrioventricular pathways well away from the septum), these limbs commonly have "atrioventricular node like" properties. ${ }^{34}$ It is this characteristic that gives adenosine the ability to terminate tachycardia in either limb of these reentrant forms of tachycardia.
1 Overholt ED, Rheuban KS, Gutgesell HP, Lerman BB, DiMarco JP. Usefulness of adenosine for arrhythmias in DiMarco JP. Usefulness of adenosine for arrhythm

2 Till J, Shinebourne EA, Rigby ML, Clarke B, Ward DE, treatment of supraventricular tachycardia in infants and children. Br Heart $J$ 1989;62:204-11. Smith RT, Sellers TD, DiMarco JP. Differential electrophysiologic properties of decremental retrograde pathways of long RP' tachycardia. Circulation 1987;76:21-31.

4 Wienecke MN, Case CL, Gillette PC. Adenasine's effectiveness in long RP reentrant tachycardia: additional evidence of the decremental qualities of the retrograde limb. Clin Cardiol 1992;15:114-6. In: Gillette PC, Garson A, eds. Pediatric arrhythmias: electrophysiology and pacing. Philadelphia: WB Saunders 1990:408-20.

6 Bharati S, Moskowitz WB, Scheinman M, Estes M, Lev $M$. Junctional tachycardias: anatomic substrate and its significance in ablative procedures. J Am Coll Cardiol 1991;18:179-86. Rowland E. Efficacy and safety of adenosine in the

3 Lerman BB, Greenberg M, Overholt ED, Swerdlow CD,

5 Ludomirsky A, Garson A: Supraventricular tachycardia. 